\title{
Role of Brahmi and Ashwagandha in Anti-Ageing
}

\author{
Nishant Patnaik \\ BiochemistryBlog.com, NeurophysiologyBlog.com, Varanasi, India \\ Email: patnaik639@yahoo.com
}

Received 30 September 2015; accepted 18 October 2015; published 21 October 2015

Copyright (C 2015 by author and Scientific Research Publishing Inc.

This work is licensed under the Creative Commons Attribution International License (CC BY). http://creativecommons.org/licenses/by/4.0/

(c) (i) Open Access

\begin{abstract}
Aging is mostly associated with reduction in cognitive abilities even in the presumed healthy. The rise in the aging population across various countries is a representation of an emerging public health challenge in the perspective of disability and the impact on the economy. Safe medicines that improve cognition abilities in elderly people may arrest the personal and social losses caused by cognitive decline. An effective way of combating stress is by use of adaptogen such as Brahmi and Ashwagandha, which are safe and effective stress relievers. This paper seeks to analyze the efficiency and safety of highly concentrated, full spectrum extracts of Brahmi and Ashwagandha roots in anxiety and stress reduction and in enhancing the overall well-being of stressed adults.
\end{abstract}

\section{Keywords}

Cognition, Aging Population, Public Health, Stress

\section{Introduction}

The best way to manage anxiety and panic attack would be through a holistic treatment approach which entails checking the food we intake, exercising, and living a healthy life. Nonetheless, sometimes living a healthy life is never enough and one may still be susceptible to these diseases. Numerous individuals run to prescription or over the counter (OTC) drugs to tackle this problem. However, there is always a better and safer alternative in the name of natural herbal anxiety supplements, based on ancient medicinal knowledge. A doctor's visit to address anxiety or panic will almost always result in prescription of antidepressants (SSRIs) or a group of sedative drugs by the name benzodiazepines. Though both can be effective, they have serious drawbacks such as sexual dysfunction, dependence/addiction, weight gain, and a host of health and cognitive complications. The worst part is when you realize the side effects are unpleasant for you and would want to cease taking a benzodiazepine or SSRI; withdrawal symptoms are always the price you have to pay. Although you may get psychological 
addiction to these drugs, chances are high that your body may become dependent. However, there are a number of anxiety herbs that give one real relief devoid of the harsh side effects of or addiction risk. Such are the Brahmi (Bacopa monnieri) [1] and Ashwagandha (Withania somnifera/Indian ginseng).

\section{Role of Ashwagandha (Withania somnifera/Indian Ginseng) in Anti-Ageing}

\subsection{What Is Ashwagandha?}

Ashwagandha is botanically called Withania somnifera Dunal and is a member of the Solanaceaae family. It is commonly called Indian Ginseng or Winter Cherry. The name "Ashagwandha" literally means "smell of horse" since the herb's fresh roots smell like horse and the fact that one who takes the herb extracts may develop the vitality and strength of a horse [2]. It has multifarious rejuvenative effects on the human body and thus referred to as a "royal herb". The herb is multi-purpose in nature and acts on different body systems such as the immune system, neurological system, the endocrinal system, the energy-production system, and the reproductive system.

\subsection{Adaptogen}

Extensive studies have been done on adaptogen due to its popularity. It has been studied as an antioxidant, adaptogen, anxiplytic, anticancer, antidepressant, thyroid modulating, cardio-protective, immunomodulating, antifungal, antibacterial, neuro-protective, anti-inflammatory, hematopoietic and cognitive enhancing agent. Ashwagandha is made up of a variety of constituents like sitoindoside, withanolides, among other alkaloids that are medicinally and pharmacologically vital since they protect cells from oxidative damage and disease. A series of tests conducted to establish the anti-stress qualities of sitoindoside VII and sitoindoside VIII deduced that both portrayed anti-stress activity. Rats were also used to test sitoindoside IX and X for immunomodulatory and central nervous system effects that impact on memory, stress, and learning [1]. Stress-induced gastric ulcer was also slightly reduced by the sitoindoside IX and X.

\section{Role of Brahmi (Bacopa monnieri) in Anti-Ageing}

\subsection{Importance of Brahmi for Pharmacologists and Phytotherapists}

Bacopa monnieri has been used over time in Ayurvedic medicine, be it alone or together with other herbs, to boost memory and learning, as a sedative, and an anti-epileptic [3]. Its English name is water hyssop and is a famous aquarium plant that is normally grown in wet tropical environments. Pharmacologists and phytotherapists have grown a lot of interest in this plant and a survey conducted in Australia showed it to be the most popular memory enhancer among Australian consumers of ages 60 - 64 [4].

\subsection{Motor Enhanced Learning and Enhancing Mental Capability}

B. monnieri entire plant or alcohol extracts has been tested on animals and has showed results of enhanced motor learning and grasping, memory consolidation, and retention in rats. Saponins (bacopasides, bacosides, or bacopasapoinins) have been credited with the memory-enhancing abilities of B. monnieri. Bacopa saponins constituents have been proven to enhance mental retention that triggers avoidance response in rats, as well as reversing amnesic effects of scopolamine, neurotoxin, electroshock, phenytoin, and immobilization stress [5]. Hypothesized action mechanisms include y-aminobutyric acid-ergic modulation, cholinergic upregulation, brain protein synthesis, antioxidant effects, 5-HT agonism, and brain stress hormones modulation. Extracts from Bacopa have also ameliorated neurotoxin effects of aluminum and nicotine. It has also lowered the levels of Beta-amyloid in the brain of a doubly transgenicmodel of quick amyloid deposition (PSAPP mice), pointing to action mechanisms relevant to Alzheimer's disease [6].

\section{Conclusion}

Highly concentrated, full spectrum extracts of Brahmi and Ashwagandha roots can be safely used in anxiety and stress reduction and in enhancing the overall well-being of stressed adults, thus its adaptogen abilities. Furthermore, B. monnieri extracts have the ability to safely boost cognitive abilities during aging. However, there is still room for research to explore what mechanism $B$. monnieri employs to achieve the results; be it working on brain 
chemistry to affect motor processes, or reducing the performance-degrading impacts of arousal on difficult tasks, or increasing tolerance to frustration, or some other mechanism.

\section{References}

[1] Alexander, P. and Georg, W. (2009) Evidence-Based Efficacy of Adaptogens in Fatigue, and Molecular Mechanisms Related to their Stress-Protective Activity. Current Clinical Pharmacology, 4, 198-219. http://dx.doi.org/10.2174/157488409789375311

[2] Singh, S. and Sushil, K. (1998) Withania somnifera: The Indian Ginseng Ashwagandha. Central Institute of Medicinal and Aromatic Plants, Lucknow. http://dx.doi.org/10.1021/np020238w

[3] Russo, A. and Borrelli, F. (2005) Bacopa monniera, a Reputed Nootropic Plant: An Overview. Phytomedicine: International Journal of Phytotherapy and Phytopharmacology, 12, 305-317. http://dx.doi.org/10.1016/j.phymed.2003.12.008

[4] Vijayan, V. and Helen, A. (2007) Protective Activity of Bacopa monniera Linn. on Nicotine-Induced Toxicity in Mice. Phytotherapy Research: PTR, 21, 378-381.

[5] Hou, C.-C., Lin, S.-J., Cheng, J.-T. and Hsu, F.-L. (2002) Bacopaside III, Bacopasaponin G, and Bacopasides A, B, and C from Bacopa monniera. Journal of Natural Products, 65, 1759.

[6] Peng, L., Zhou, Y., Kong, D.Y. and Zhang, W.D. (2010) Antitumor Activities of Dammarane Triterpene Saponins from Bacopa monniera. Phytotherapy Research, 24, 864-868. 